\title{
Epigenome-wide meta-analysis of blood DNA methylation and its association with subcortical volumes: findings from the ENIGMA Epigenetics Working Group
}

\author{
Tianye Jia $\mathbb{1}^{1,2,3}$ et al.
}

Received: 30 March 2019 / Revised: 9 November 2019 / Accepted: 12 November 2019 / Published online: 6 December 2019

(c) The Author(s) 2019. This article is published with open access

\begin{abstract}
DNA methylation, which is modulated by both genetic factors and environmental exposures, may offer a unique opportunity to discover novel biomarkers of disease-related brain phenotypes, even when measured in other tissues than brain, such as blood. A few studies of small sample sizes have revealed associations between blood DNA methylation and neuropsychopathology, however, large-scale epigenome-wide association studies (EWAS) are needed to investigate the utility of DNA methylation profiling as a peripheral marker for the brain. Here, in an analysis of eleven international cohorts, totalling 3337 individuals, we report epigenome-wide meta-analyses of blood DNA methylation with volumes of the hippocampus, thalamus and nucleus accumbens (NAcc)—three subcortical regions selected for their associations with disease and heritability and volumetric variability. Analyses of individual CpGs revealed genome-wide significant associations with hippocampal volume at two loci. No significant associations were found for analyses of thalamus and nucleus accumbens volumes. Cluster-based analyses revealed additional differentially methylated regions (DMRs) associated with hippocampal volume. DNA methylation at these loci affected expression of proximal genes involved in learning and memory, stem cell maintenance and differentiation, fatty acid metabolism and type- 2 diabetes. These DNA methylation marks, their interaction with genetic variants and their impact on gene expression offer new insights into the relationship between epigenetic variation and brain structure and may provide the basis for biomarker discovery in neurodegeneration and neuropsychiatric conditions.
\end{abstract}

\section{Introduction}

Structural brain measures are important correlates of developmental and health outcomes across the lifetime. A large body of evidence has revealed age-related reductions in grey matter structures across the brain [1], notably in the hippocampus, which correlates with declining memory performance in older adults [2, 3]. Recent findings from large-scale neuroimaging analyses within the ENIGMA

These authors contributed equally: Paul M. Thompson, Gunter Schumann

Supplementary information The online version of this article (https:// doi.org/10.1038/s41380-019-0605-z) contains supplementary material, which is available to authorized users.

Sylvane Desrivières

sylvane.desrivieres@kcl.ac.uk

Extended author information available on the last page of the article. consortium have revealed consistent patterns of cortical $[4,5]$ and subcortical [5-8] brain volume reductions across several neuropsychiatric disorders. Of all structures reported, the hippocampus was the most consistently and robustly altered, being smaller in major depressive disorder [6], schizophrenia [7], attention deficit hyperactivity disorder [8], obsessive-compulsive disorder (OCD) [9], and posttraumatic stress disorder [10]. Other notable changes included volume reductions in the thalamus and nucleus accumbens (NAcc) in schizophrenia [7, 8], as well as volume increases in the same regions in paediatric OCD [9].

Such differences in brain structure may fundamentally reflect the effects of genetic and environmental factors and their interplay, as suggested by the study of discordant monozygotic twins [11]. DNA methylation is an epigenetic mechanism that may underlie gene-environment contributions to brain structure. It is under the influence of genetic [12, 13] and developmental [13-15] factors and plays an important role in brain development 
and disease, by regulating gene expression. DNA methylation is also a mechanism through which external stimuli, such as the environment, may contribute to expression of common diseases such as neurodegenerative disorders [16].

While efforts to identify genetic factors influencing brain structure have flourished in recent years [17-19], epigenetic studies of brain-related phenotypes remain very sparse. A considerable constraint is the need for a surrogate tissue for epigenetic studies of the living human brain. Crucially, while initial reports have demonstrated that although DNA methylation patterns are largely tissue-specific, often differing between blood and brain $[20,21]$, there are also similarities [22] and blood DNA methylation shows promise as a biomarker for brain-related traits, including neuropsychiatric disorders [23-27], cognitive ability [28, 29] and future psychopathology [26]. However, only a few studies of small sample sizes have reported associations between blood DNA methylation and brain phenotypes [26, 30-32].

Here, we built upon these findings and performed a large multisite epigenome-wide association study (EWAS) of structural brain volumes in 3337 individuals from 11 cohorts. We focussed on analyses of the hippocampus, thalamus and NAcc, based on relevance of these subcortical brain regions for disease and on heritability of these phenotypes. We selected the hippocampus as the brain structure most consistently and robustly altered in neuropsychiatric disorders, as described above. We also selected the thalamus and NAcc as subcortical regions with the highest and lowest twin-based heritability estimates, respectively $[18,33]$, to test a model according to which a substantial fraction of the heritability of complex traits may be due to epigenetic variation [34].

\section{Material and methods}

\section{Subjects and brain measures}

The brain phenotypes examined in this study are from the ENIGMA analysis of high-resolution MRI brain scans of volumetric measures (full details in [18]). Our analyses were focussed to mean (of left and right hemisphere) volumetric measures of three subcortical areas: the hippocampus, thalamus and NAcc, selected for their link to disease, different levels of heritability, and developmental trajectories. MRI brain scans and genome-wide DNA methylation data were available for 3337 subjects from 11 cohorts (Supplementary Table 1). All participants in all cohorts in this study gave written informed consent and sites involved obtained approval from local research ethics committees or Institutional Review Boards.

\section{DNA methylation microarray processing and normalization}

Blood DNA methylation was assessed for each study using the Illumina HumanMethylation450 (450k) microarray, which measures $\mathrm{CpG}$ methylation across $>485,000$ probes covering $99 \%$ of RefSeq gene promoters [35], following the manufacturer's protocols. Standardised quality control procedures and quantile normalization were performed using the $\operatorname{minfi}$ Bioconductor package in R [36]. Please refer to Supplementary Materials and Methods for more details.

\section{Epigenome-wide association analysis}

EWAS with volumes of the thalamus, hippocampus and NAcc were performed for each site separately with standardised procedures (see Supplementary Materials and Methods for details), where control variables included sex, age, age [2], intracranial volume, methylation composition (the first four components), blood cell-type composition (the first two components), sample batches (when applicable), recruitment centres (when applicable) and disease status (when applicable). For studies with data collected across several centres, dummy-coded covariates were also included in the model.

Results from each cohort were then meta-analysed by combining correlations (fisher's r-to-Z transformed) across all 11 cohorts with a fixed effect model, weighted by the corresponding inversed variance [37]. False discovery rates (FDR) were computed (correcting for the number of brain regions tested and the number of DNA methylation probes) and FDR $<0.05$ was considered statistically significant. Differentially methylated regions (DMRs) were identified by applying the Comb-p algorithm [38] (Supplementary Materials and Methods).

\section{Results}

\section{Associations of DNA methylation with subcortical volumes: analyses of individual CpG sites}

We first investigated the association of DNA methylation at individual $\mathrm{CpG}$ sites in whole blood samples with the mean bilateral volumes of the hippocampus, thalamus and NAcc. Meta-analysis was applied by combining correlations across all 11 cohorts with a fixed effect model, weighting for sample size. We identified two CpGs associating with the volume of the hippocampus (Fig. 1a; Supplementary Table 2) at an experiment-wide (correcting for the number of brain regions tested) FDR $<0.05$. The analyses of thalamus and NAcc volumes identified no $\mathrm{CpG}$ reaching the experiment-wide FDR threshold. Q-Q plots for the $P$ values of the analyses showed no evidence of $P$ value inflation. 


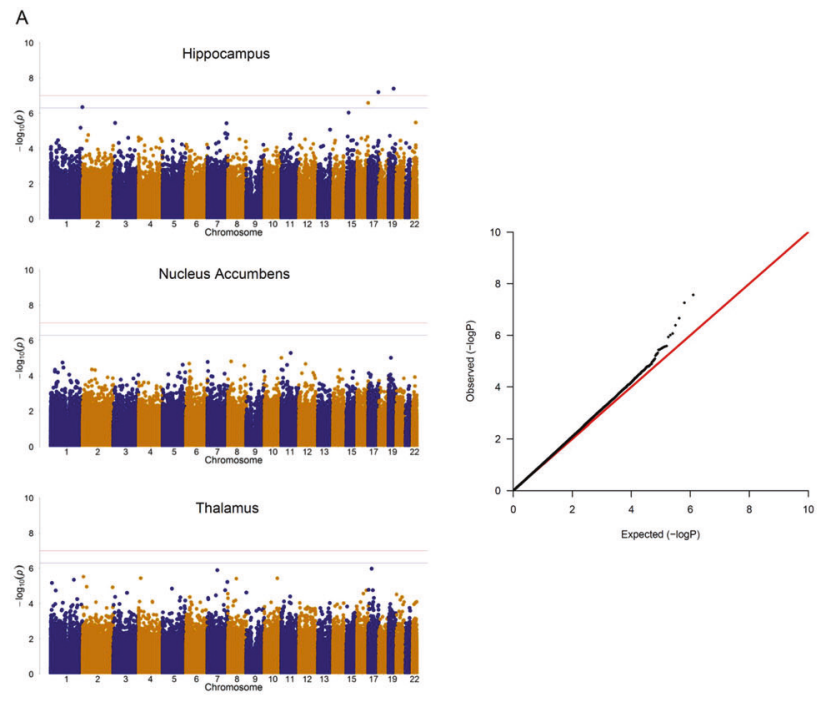

Fig. 1 a Manhattan plots (left) summarizing the association results for the hippocampus, thalamus and NAcc volumes. The red and blue lines represent the genome-wide FDR significance level (corrected for three brain regions) and non-corrected FDR significance level, respectively. Quantile-quantile plots (right) of multivariate GWAS of all traits (volumes of the hippocampus, thalamus and accumbens) show that the observed $P$ values only deviate from the expected null distribution at the most significant values, indicating no undue inflation of the results. b Forest plots show the effect (i.e. correlations between $\mathrm{CpG}$ methylation and hippocampus volume) at each of the contributing sites to the

The CpGs associated with hippocampal volume explained each $0.9 \%$ of the phenotypic variance. Their effects were consistent across cohorts, with similar effect sizes for the $\operatorname{cg} 26927218$ site $(P>0.1$, Cochran's $Q$ test), while moderate heterogeneity in the magnitude, but not the direction of effects was noted for cg17858098 (Fig. 1b). Effect sizes for analyses with and without patients across the 11 cohorts were very highly correlated $(r \geq 0.99)$ for CpGs with $P<1 \times 10^{-3}$, indicating that these effects were unlikely driven by disease. These $\mathrm{CpGs}$ were annotated to the brain-specific angiogenesis inhibitor 1-associated protein 2 (BAIAP2) gene (also known as IRSp53; cg26927218) - encoding a synaptic protein whose expression in the hippocampus is required for learning, memory [39] and social competence [40] and to the enoyl-CoA hydratase-1 (ECH1; cg17858098), which encodes an enzyme involved in the $\beta$-oxidation of fatty acids [41].

CpGs associated with hippocampal volume showed effects specific for this structure rather than pleiotropic effects. Of the $340 \mathrm{CpGs}$ associated with hippocampus volume at $P<5 \times 10^{-4}$ (Supplementary Table 2), $71 \%$ were associated only with the hippocampus, $21 \%$ were shared with the thalamus and few with the NAcc (Fig. 1c). These closer epigenetic links between hippocampus and thalamus
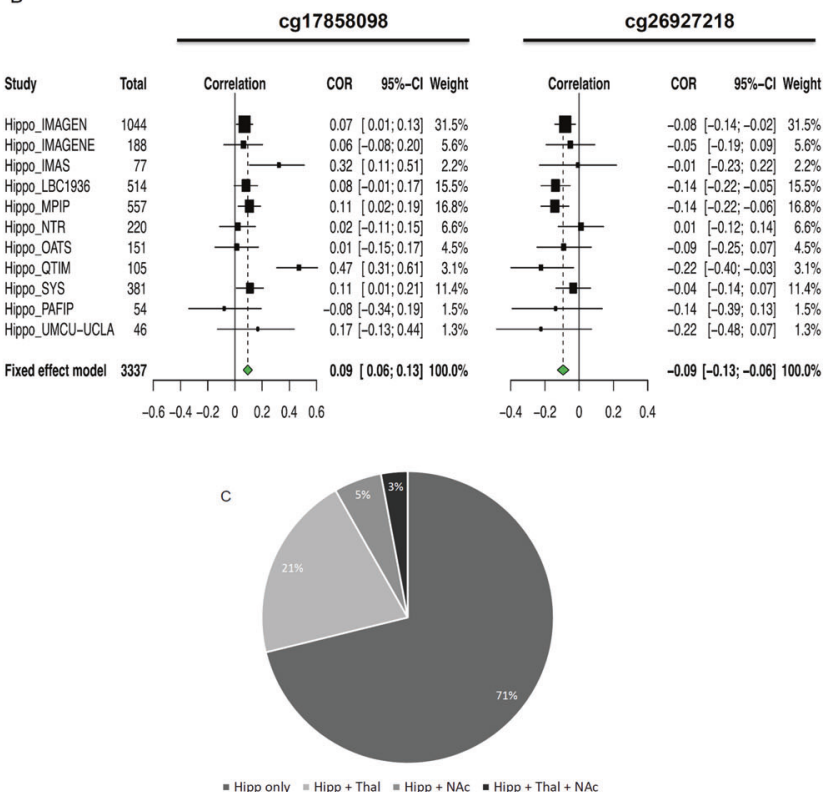

meta-analysis. The size of the dot is proportional to the sample size, the correlation level is shown on the $x$-axis, and confidence interval is represented by the line. c Pie chart of distribution of the $340 \mathrm{CpGs}$ associated with hippocampus volume at $P<5 \times 10^{-4}$. The chart indicates the proportion of these $\mathrm{CpG}$ sites that are unique to the hippocampus or that are also associated (nominally, at $p<0.05$ ) with the two other volumetric phenotypes investigated. In general, $\mathrm{CpGs}$ that influence other phenotypes than hippocampus volume have higher effect on thalamus than on NAcc volume

reflected closer correlations between their volumes $\left(r_{H^{*} T}=\right.$ $0.367, P=5.78 \times 10^{-34}$ and $r_{H_{N}}=0.201, P=8.36 \times 10^{-11}$, for correlations of hippocampal volumes with thalamus and NAcc volumes, respectively).

\section{Associations of DNA methylation with subcortical volumes: differentially methylated regions}

The analyses described above did not account for effects of DNA methylation clusters at regions formed by spatially correlated $\mathrm{CpGs}$, which often occur within regulatory regions in the genome and are powerful means to control gene expression. Therefore, in the following analyses, we set out to identify such DNA methylation clusters (i.e. differentially methylated regions, DMRs) by applying the comb-p algorithm [38] to our epigenome-wide meta-analyses of hippocampal volume (see Supplementary Materials and Methods). Several DMRs significantly associated with the volume of hippocampus in the meta-analysed results (Šidák [42] corrected $P<0.05$, number of consecutive probes $\geq \mathrm{E} 2$; total numbers of DMRs $=20$; Table 1). A DMR that included the $\operatorname{cg} 26927218$ site was identified $\left(P_{\text {corrected }}=9.44 \times 10^{-4}\right)$, further supporting the association of BAIAP2 methylation with hippocampal volume. In addition to being identified from the 


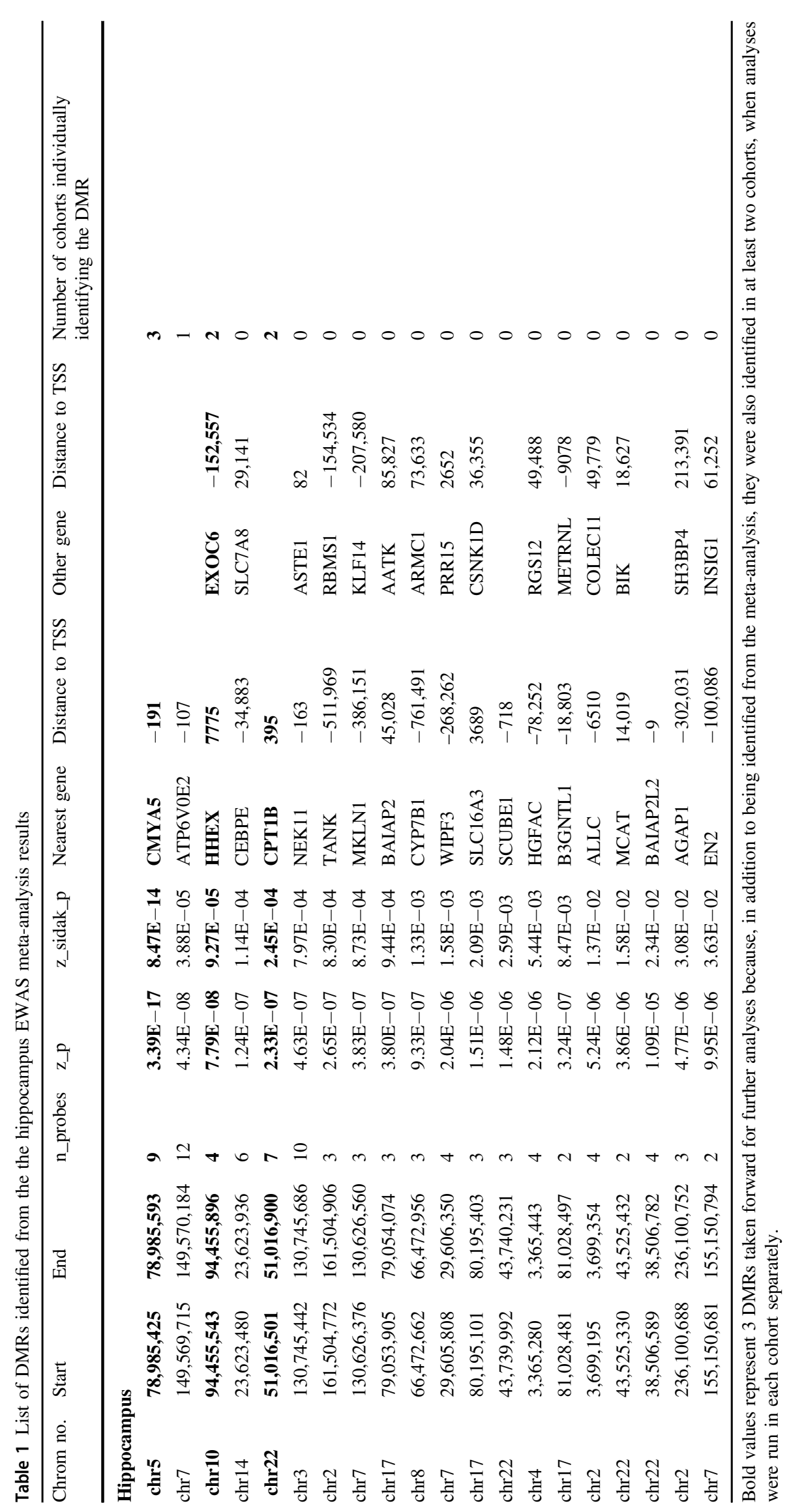


meta-analysed data, three of these DMRs were identified in at least two cohorts, when analyses were run on EWAS results of each cohort separately, indicating that their association with brain volumes were unlikely to be due to chance. They were located within the cardiomyopathy associated gene 5 (CMYA5; $P_{\text {corrected }}=8.47 \times 10^{-14}$; this DMR is subsequently referred to as DMR1), encoding an expression biomarker for diseases affecting striated muscle [43-46] and possibly a schizophrenia risk gene [47]; the hematopoietically expressed homeobox (HHEX; $P_{\text {corrected }}=9.27 \times 10^{-5}$; DMR2) gene, encoding a homeobox transcription factor controlling stem cells pluripotency and differentiation in several tissues [48-52], and a well-known risk loci for type 2 diabetes [53], as well as the carnitine palmitoyltransferase 1B (CPT1B; $P_{\text {corrected }}=2.45 \times 10^{-4}$; DMR3) gene, encoding a ratelimiting enzyme in the mitochondrial beta-oxidation of long-chain fatty acids, whose expression enhances reprogramming of somatic cells to induced pluripotent stem cells [54], cancer cell self-renewal and chemoresistance [55]. There was a significant degree of correlation of DNA methylation at these DMRs $\left(r=0.155, P=7.30 \times 10^{-8}\right.$ and $r=0.147, P=$ $2.91 \times 10^{-7}$, for DMR1 versus DMR3 and DMR1 versus DMR2, respectively). These three DMRs were also taken forward for further analyses.

\section{Effects of differential methylation on gene expression}

We measured the impact of DNA methylation on expression of neighbouring genes (cis-effects) in 631 IMAGEN subjects for which DNA methylation and mRNA expression data were available (see Supplementary Materials and Methods). Methylation at most loci affected gene expression, with the effects of DMRs being larger than that of individual CpGs (i.e. cg26927218). Several isoforms are expressed from BAIAP2, and isoform-specific effects were observed for cg26927218; methylation at this locus correlated with increased expression of the short isoform for BAIAP2 ( $\beta=0.016, P=5 \times 10^{-3}$; Fig. 2a). There were no significant effects of cg17858098 on ECH1 mRNA levels $(\beta=-0.008, P=0.201)$. Given the correlations between the selected three DMRs noted above, we controlled for methylation at the other two DMRs when testing for effects of a given DMR on gene expression. As shown in Fig. 2b, DMR1 methylation had no effect on expression of CMYA5 ( $\beta=-0.227, P=0.492)$, tending instead to have contrasting effects on expression of neighbouring genes $(\beta=$ $-0.410, P=0.039$ and $\beta=0.554, P=0.019$ for PAPD4 and MTX3, respectively). Methylation at DMR2 increased expression of its closest gene, HHEX $(\beta=0.351, P=$ 0.020). Methylation at DMR3 had strong effects on expression of the adjacent $C P T 1 B$ gene $(\beta=1.670, P=$ $\left.2.55 \times 10^{-59}\right)$. Trans-effects were also noted for this
DMR, as it associated with increased expression of PAPD4 $\left(\beta=0.724, P=1.21 \times 10^{-7}\right)$, a gene adjacent to DMR1.

\section{Correlations of DNA methylation between blood and brain}

To investigate if the above findings would remain relevant for the brain, we first compared methylation levels at the selected differentially methylated loci (i.e. two $\mathrm{CpG}$ sites and three DMRs) in blood and brain tissues sampled from the same individuals to establish the degree to which blood methylation levels at selected loci correlated with their brain methylation patterns. Then, we compared the degree of these blood-brain covariations (i.e. the extent to which of DNA methylation in blood correlated with DNA methylation in brain) to the corresponding $Z$-values from the hippocampal EWAS. We evaluated these effects across all three DMRs, as well as within each DMR. It is important to point out that higher degree of blood-brain covariations in methylation, which indicates a higher proportion of shared information between blood and brain, would result in increased strength in association between blood DNA methylation and hippocampus volume, solely if this association was indeed mediated by brain DNA methylation. Please see Supplementary Materials and Methods for details of the approach.

We compared methylation levels at these sites in blood and brain tissues (prefrontal cortex, entorhinal cortex, superior temporal gyrus and cerebellum) sampled from the same individuals $(N=75)$ using the blood-brain DNA methylation comparison tool [56] (see Supplementary Materials and Methods; Supplementary Table 3). There was no significant correlation between blood and brain methylation levels at the individual $\mathrm{CpG}$ sites (cg26927218-BAIAP2 and $\operatorname{cg} 17858098-E C H 1)$. On the other hand, interindividual variation in whole blood was a moderate predictor of interindividual variation in all tested cortical brain areas for DMR1 and DMR3 (strongest correlations: $r=0.54, P=1.20 \times 10^{-6}$ and $r=0.59$, $P=2.37 \times 10^{-8}$, respectively; Supplementary Table 3 ). For DMR2, correlations were more varied with the strongest correlation in the superior temporal gyrus $(r=$ $0.37, P=9.68 \times 10^{-4}$; Supplementary Table 3 ). Generally, correlations were stronger in cortical brain regions than in the cerebellum. Crucially, the degree of blood-brain covariations in DNA methylation at these sites predicted associations between blood DNA methylation and hippocampus volume with moderate to large effect sizes (Supplementary Table 4 and Supplementary Fig. 1). These effects were particularly notable in the superior temporal gyrus, across all three DMRs $(r=0.751, t=$ $4.54, \mathrm{df}=16, P_{\text {one tailed }}=6.6 \times 10^{-3}$ after Bonferroni correction for four brain regions), and at each DMR ( $r=$ $0.707, t=2.65, \mathrm{df}=7, P_{\text {one tailed }}=0.017$ for DMR $1 ; r=$ 


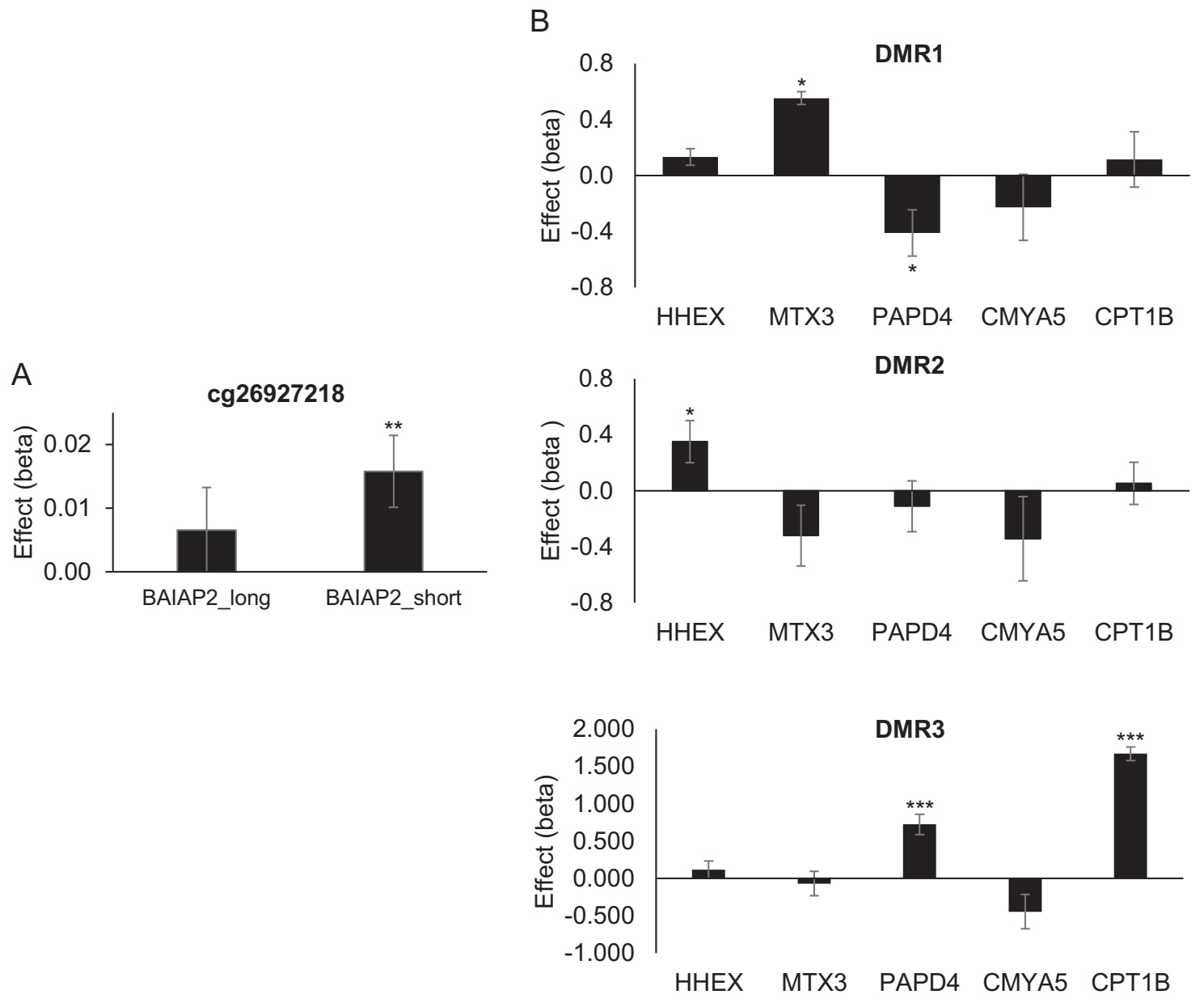

Fig. 2 Analyses of top CpG (a) and DMRs (b) demonstrate effects of DNA methylation on gene expression in 631 subjects from the IMAGEN sample. In the DMR analyses, linear regression analyses tested relationship between methylation at the listed DMR and

$0.964, t=5.14, \mathrm{df}=2, P_{\text {one tailed }}=0.018$ for DMR2; $r=$ $-0.748, t=-2.52, \mathrm{df}=5, P_{\text {one tailed }}=0.027$ for DMR3). There was also moderate prediction by the degree of covariation of DNA methylation in prefrontal cortex, across all three DMRs $(r=0.417, t=1.84, \mathrm{df}=16$, $\left.P_{\text {one tailed }}=0.042\right)$, and with DMR2 $(r=0.966, t=5.26$, $\left.\mathrm{df}=2, P_{\text {one tailed }}=0.017\right)$. These results strongly suggest that associations between hippocampal volume and blood DNA methylation levels at the selected DMRs are largely mediated by their DNA methylation levels in the brain (see Supplementary Materials and Methods for more details).

Another comparison between methylation in blood and other brain regions-Brodmann area (BA)7 (parietal cortex); BA10 (anterior prefrontal cortex) and BA20 (ventral temporal cortex) - using a smaller dataset of 16 BECon post-mortem samples [57] revealed similar patterns (see Supplementary Materials and Methods; Supplementary Fig. 2). For DMR1, there were moderate correlations expression of $H H E X, M T X 3, P A P D 4, C M Y A 5$ and $C P T 1 B$, controlling for methylation at the other two DMRs. Results represent unstandardized coefficients \pm S.E.M. $* p<0.05 ; * * p<0.01 ; * * * p<0.001$

between blood and BA7 methylation at all CpGs $(r=$ 0.13-0.47) and between blood and BA10 for most CpGs $(r=0.13-0.30)$. For DMR3, correlations between blood and brain methylation were strong in all areas $(r=$ 0.37-0.86), while the degree of correlations varied at DMR2 ranging from -0.35 to 0.34 , depending on the $\mathrm{CpG}$ site and the brain area.

\section{Genetic contributions to differential DNA methylation associated with hippocampal volume}

Given that genetic factors may underlie the correlations between DNA methylation in different tissues, we searched for methylation QTLs in two datasets. A search in the ARIES mQTL database [58] identified several SNPs associated with methylation at the DMR1 and DMR3 loci (see Supplementary Materials and Methods; Supplementary Table 5A). The strongest mQTLs, rs131758 and rs4441859, affected methylation such that the A-allele at these SNPs associated with 
increased methylation at DMR3 and DMR1, respectively. These effects were replicated in two other datasets [59, 60] (see Supplementary Materials and Methods; Supplementary Table 5B). Remarkably, eQTL analyses indicated that these alleles correlated with expressions of CMYA5 and CPT1B, albeit differently. While the effects of the rs4441859_A allele were tissue-specific, the rs131758_A allele increased CPT1B expression in all tissues, including the brain (Supplementary Table 5C and Supplementary Fig. 3).

Furthermore, we considered whether there was a significant overlap between DNA methylation differences identified in this study and SNPs associated with hippocampal volume. To test this, we used the recent genome-wide association studies of hippocampal volume conducted by ENIGMA [18] (excluding the IMAGEN data; GWAS association thresholds $P<5 \times 10^{-6}$ and $P<5 \times 10^{-7}$ ) as a dataset for significant hippocampal SNP regions, adapting MAGENTA [61] (see Supplementary Materials and Methods), the gene setsbased enrichment analysis tool for GWAS data to the analysis of methylation data. SNPs were merged into genomic regions that were then examined for overlap with DNA methylation identified in hippocampal EWAS performed in the IMAGEN sample. These analyses revealed a significant overlap between DNA methylation loci and SNP loci influencing hippocampal volume (Supplementary Table 6).

\section{Discussion}

In this large epigenome-wide meta-analysis we identified for the first time differentially methylated $\mathrm{CpG}$ sites and genomic regions whose levels of DNA methylation correlate with variation in hippocampal volume. We further demonstrate the potential of using blood to discover epigenetic biomarkers for the living human brain. Methylation at these sites affect the expression of genes required for hippocampal function and metabolic regulation. At the identified sites, the observation that DNA methylation variation in blood can mirror that of brain tissues, and that the degree of this covariation could predict the association of blood DNA methylation with hippocampus volume, helps us generate hypotheses as to how modifiable factors such as diet and lifestyle may contribute to some of the impairments associated with diabetes and neurodegenerative conditions [62].

Changes in hippocampal volumes are hallmarks of brain development predictive of cognitive deficits generally associated with aging and neurodegeneration. While large hippocampal volume is linked with good memory and cognitive function, hippocampal atrophy is associated with the development of a range of neurodegenerative [63] and neuropsychiatric disorders [6-8, 10]. Modifiable factors such as obesity, exercise, stress and medication can reduce or increase the size of the hippocampus throughout life [63].
Collectively, our findings support these observations, pointing to associations of hippocampal volume with fatty acid metabolism, as discussed below.

Two of the top hits identified ( $C P T 1 B$ and $E C H 1)$ encode key enzymes involved in $\beta$-oxidation of fatty acids. These enzymes act on the same pathway, CPT1B being necessary for the transport of long-chain fatty acids into the mitochondria and ECH1 for a key step in their $\beta$-oxidation. Fatty acids (notably the omega-3 polyunsaturated fatty acids) benefit brain development and healthy brain aging by modulating neurogenesis and protecting from oxidative stress throughout the lifespan [64]. More specifically, neural precursors in the hippocampus and subventricular zone require fatty acid oxidation for proliferation [65]. This led to the proposition that abnormalities in brain lipid metabolism contribute to hippocampal dysfunction in $\mathrm{AD}$ by their ability to suppress neurogenesis at early stages of disease pathogenesis [66]. Accordingly, fatty acid metabolism in the brain seems to be closely related to the pathogenesis of Alzheimer's disease [67].

Further links between metabolism and hippocampal volume were suggested by our identification of a region annotated to a replicated risk locus for T2D (HHEX) [53]. The metabolic alterations observed in T2D may induce cognitive dysfunction [68] by exacerbating declines in hippocampal volumes associated with aging [69] and AD pathology [70], a process to which HHEX may contribute [71]. This is supported by findings that genetic variations within the HHEX gene region may underlie the association of T2D with AD, with the HHEX rs1544210_AA genotype interacting with diabetes to increase the risk of dementia and AD by more than fourfold [71]. Furthermore, individuals with diabetes who carry the HHEX rs1544210_AA genotype tend to have significantly smaller hippocampal volumes than those without these conditions [71].

DNA methylation at most loci had clear, albeit distinct effects on gene expression. Notable transcript-specific effects were observed for cg26927218 on BAIAP2. The cg26927218 locus is located in a DNase I hypersensitive site, characteristic of regions actively involved in transcriptional regulation [72], within a consensus DNA binding sequence for the MYC associated factor X (MAX)-a transcription factor controlling cell proliferation, differentiation, and apoptosis. MAX belongs to a class of transcription factors that recognize CpG-containing DNA binding sequences, only in their unmethylated form [73, 74]. Thus, methylation at cg26927218 may affect expression of the BAIAP2 short variant by directly interfering with the function of this transcription factor. A role for the region surrounding cg26927218 in transcriptional regulation is further supported by findings showing that a genetic variant (rs8070741) near cg26927218 enhances cortical expression of the BAIAP2 short variant [75]. 
Besides the hippocampus, none of the other two subcortical structures investigated generated significant results. This may reflect a unique role of the hippocampus in brain development, possibly related to it being a site of neurogenesis. These findings are also consistent with the relative heritability of the different subcortical structures, indicating higher twin-based heritability estimates for larger (hippocampus and thalamus) compared with smaller (NAcc) subcortical structures but overall low SNP-based heritability [18]. This supports the model according to which a substantial fraction of the heritability of complex traits is due to epigenetic variation [34]. Our analyses on genetic contributions to DMRs' effects also suggest that epigenetic control is partially modulated by genetic variations, which is further suggested by the overlap between GWAS and EWAS of hippocampal volume.

In conclusion, we have identified DNA methylation at several loci that correlate with hippocampus volume, which suggest for the first time possible biological pathways by which modifiable and metabolic factors might contribute to the pathology of neurodegenerative diseases. A clear limitation is the small number of cohorts for which both MRI and DNA methylation data are available, we nonetheless provide a rigorous roadmap that should encourage larger and more extensive future studies. We also acknowledge several other limitations, such as the shortage of datasets enabling direct comparison of blood and brain DNA methylation patterns. In particular, the lack of datasets including the hippocampus as a brain region prevented us from establishing a direct link between DNA methylation in blood and in the hippocampus. However, we do provide evidence showing that DNA methylation in the superior temporal gyrus mediates associations between blood DNA methylation and hippocampal volume. Also, given the cross-sectional nature of this study, none of the associations identified can be claimed to be causal. Nevertheless, our work demonstrates the usefulness of combining peripheral DNA methylation markers and neuroimaging measures for biomarker discovery in common neurological and neuropsychiatric conditions.

\section{Code availability}

The protocols used for testing association and meta-analysis and the meta-analytic results will be freely available from the ENIGMA consortium webpage upon publication (http:// enigma.ini.usc.edu/protocols/ and http://enigma.ini.usc.edu/ research/downloadenigma-gwas-results).

Author contributions Paper writing and editing: SD wrote the paper; BR, EBB, FH, GS, JL, NF, PMT, PS, SE, TC-R, TJ, TJE, TP, UB, $\mathrm{VC}, \mathrm{VF}$ and $\mathrm{ZP}$ edited the first draft; all authors critically reviewed the paper. Cohort principal investigators: AS, BC-F, DA, DIB, EBB, GS, HB, IJD, JT, LGA, MJW, MW, PRS, PSS, RAO, VC and ZP. Imaging data acquisition: AdB, DvE, EA, FN, GIZ, HF, J-LM, JJ, JL, JT, KLM, LTS, MEB, MH, PMT, PS, RB, TB, TP, VF, WC and WW. Epigenetic data acquisition: AFM, AT, DS, GB, JL, JS, JvD, JT, KAM, NGM, PS,TC-R, TJ, YL and ZP. Data analysis: AO, BR, CC, EP, JL, JS, JvD, KS, ML, NA, NJ, RJ, RR-S, SD,TC-R and TJ.

\section{Compliance with ethical standards}

Conflict of interest TB served in an advisory or consultancy role for Actelion, Hexal Pharma, Lilly, Lundbeck, Medice, Novartis, Shire. He received conference support or speaker's fee by Lilly, Medice, Novartis and Shire. He has been involved in clinical trials conducted by Shire and Viforpharma. He received royalities from Hogrefe, Kohlhammer, CIP Medien, Oxford University Press. The present work is unrelated to the above grants and relationships. HW received a speaker honorarium from Servier (2014). The other authors report no biomedical financial interests or potential conflicts of interest.

Publisher's note Springer Nature remains neutral with regard to jurisdictional claims in published maps and institutional affiliations.

Open Access This article is licensed under a Creative Commons Attribution 4.0 International License, which permits use, sharing, adaptation, distribution and reproduction in any medium or format, as long as you give appropriate credit to the original author(s) and the source, provide a link to the Creative Commons license, and indicate if changes were made. The images or other third party material in this article are included in the article's Creative Commons license, unless indicated otherwise in a credit line to the material. If material is not included in the article's Creative Commons license and your intended use is not permitted by statutory regulation or exceeds the permitted use, you will need to obtain permission directly from the copyright holder. To view a copy of this license, visit http://creativecommons. org/licenses/by/4.0/.

\section{References}

1. Salat DH, Buckner RL, Snyder AZ, Greve DN, Desikan RS, Busa $\mathrm{E}$, et al. Thinning of the cerebral cortex in aging. Cereb Cortex. 2004; $14: 721-30$.

2. Persson J, Nyberg L, Lind J, Larsson A, Nilsson LG, Ingvar M, et al. Structure-function correlates of cognitive decline in aging. Cereb Cortex. 2006;16:907-15.

3. Persson J, Pudas S, Lind J, Kauppi K, Nilsson LG, Nyberg L. Longitudinal structure-function correlates in elderly reveal MTL dysfunction with cognitive decline. Cereb Cortex. 2012;22: 2297-304.

4. Hibar DP, Westlye LT, Doan NT, Jahanshad N, Cheung JW, Ching CRK, et al. Cortical abnormalities in bipolar disorder: an MRI analysis of 6503 individuals from the ENIGMA bipolar disorder working group. Mol Psychiatry. 2018;23:932-42.

5. van Rooij D, Anagnostou E, Arango C, Auzias G, Behrmann M, Busatto GF, et al. Cortical and subcortical brain morphometry differences between patients with autism spectrum disorder and healthy individuals across the lifespan: results from the ENIGMA ASD working group. Am J Psychiatry. 2018;175:359-69.

6. Schmaal L, Veltman DJ, van Erp TG, Samann PG, Frodl T, Jahanshad N, et al. Subcortical brain alterations in major depressive disorder: findings from the ENIGMA major depressive disorder working group. Mol Psychiatry. 2016;21:806-12.

7. van Erp TG, Hibar DP, Rasmussen JM, Glahn DC, Pearlson GD, Andreassen OA, et al. Subcortical brain volume abnormalities in 2028 individuals with schizophrenia and 2540 healthy controls via the ENIGMA consortium. Mol Psychiatry. 2016;21:547-53. 
8. Hoogman M, Bralten J, Hibar DP, Mennes M, Zwiers MP, Schweren LSJ, et al. Subcortical brain volume differences in participants with attention deficit hyperactivity disorder in children and adults: a cross-sectional mega-analysis. Lancet Psychiatry. 2017;4:310-9.

9. Boedhoe PSW, Schmaal L, Abe Y, Alonso P, Ameis SH, Anticevic A, et al. Cortical abnormalities associated with pediatric and adult obsessive-compulsive disorder: findings from the ENIGMA obsessive-compulsive disorder working group. Am J Psychiatry. 2018;175:453-62.

10. Logue MW, van Rooij SJH, Dennis EL, Davis SL, Hayes JP, Stevens JS, et al. Smaller hippocampal volume in posttraumatic stress disorder: a multisite ENIGMA-PGC study: subcortical volumetry results from posttraumatic stress disorder consortia. Biol Psychiatry. 2018;83:244-53.

11. Suddath RL, Christison GW, Torrey EF, Casanova MF, Weinberger DR. Anatomical abnormalities in the brains of monozygotic twins discordant for schizophrenia. N Engl J Med. 1990;322:789-94.

12. Gibbs JR, van der Brug MP, Hernandez DG, Traynor BJ, Nalls MA, Lai SL, et al. Abundant quantitative trait loci exist for DNA methylation and gene expression in human brain. PLoS Genet. 2010;6:e1000952.

13. Kaminsky ZA, Tang T, Wang SC, Ptak C, Oh GH, Wong AH, et al. DNA methylation profiles in monozygotic and dizygotic twins. Nat Genet. 2009;41:240-5.

14. Lister R, Mukamel EA, Nery JR, Urich M, Puddifoot CA, Johnson ND, et al. Global epigenomic reconfiguration during mammalian brain development. Science. 2013;341:1237905.

15. van Dongen J, Nivard MG, Willemsen G, Hottenga JJ, Helmer Q, Dolan CV, et al. Genetic and environmental influences interact with age and sex in shaping the human methylome. Nat Commun. 2016;7:11115.

16. Hwang JY, Aromolaran KA, Zukin RS. The emerging field of epigenetics in neurodegeneration and neuroprotection. Nat Rev Neurosci. 2017;18:347-61.

17. Stein JL, Medland SE, Vasquez AA, Hibar DP, Senstad RE, Winkler AM, et al. Identification of common variants associated with human hippocampal and intracranial volumes. Nat Genet. 2012;44:552-61.

18. Hibar DP, Stein JL, Renteria ME, Arias-Vasquez A, Desrivieres S, Jahanshad $\mathrm{N}$, et al. Common genetic variants influence human subcortical brain structures. Nature. 2015;520:224-9.

19. Desrivières S, Lourdusamy A, Tao C, Toro R, Jia T, Loth E, et al. Single nucleotide polymorphism in the neuroplastin locus associates with cortical thickness and intellectual ability in adolescents. Mol Psychiatry. 2015;20:263-74.

20. Davies MN, Volta M, Pidsley R, Lunnon K, Dixit A, Lovestone S, et al. Functional annotation of the human brain methylome identifies tissue-specific epigenetic variation across brain and blood. Genome Biol. 2012;13:R43.

21. Horvath S, Zhang Y, Langfelder P, Kahn RS, Boks MP, van Eijk $\mathrm{K}$, et al. Aging effects on DNA methylation modules in human brain and blood tissue. Genome Biol. 2012;13:R97.

22. Walton E, Hass J, Liu J, Roffman JL, Bernardoni F, Roessner V, et al. Correspondence of DNA methylation between blood and brain tissue and its application to schizophrenia research. Schizophr Bull. 2016;42:406-14.

23. Melas PA, Rogdaki M, Osby U, Schalling M, Lavebratt C, Ekstrom TJ. Epigenetic aberrations in leukocytes of patients with schizophrenia: association of global DNA methylation with antipsychotic drug treatment and disease onset. Faseb J. 2012;26:2712-8.

24. Lunnon K, Smith R, Hannon E, De Jager PL, Srivastava G, Volta $\mathrm{M}$, et al. Methylomic profiling implicates cortical deregulation of ANK1 in Alzheimer's disease. Nat Neurosci. 2014;17:1164-70.
25. Montano C, Taub MA, Jaffe A, Briem E, Feinberg JI, Trygvadottir R, et al. Association of DNA methylation differences with schizophrenia in an epigenome-wide association study. JAMA Psychiatry. 2016;73:506-14.

26. Ruggeri B, Nymberg C, Vuoksimaa E, Lourdusamy A, Wong CP, Carvalho FM, et al. Association of protein phosphatase PPM1G with alcohol use disorder and brain activity during behavioral control in a genome-wide methylation analysis. Am J Psychiatry. 2015;172:543-52.

27. Klengel T, Pape J, Binder EB, Mehta D. The role of DNA methylation in stress-related psychiatric disorders. Neuropharmacology. 2014;80:115-32.

28. Marioni RE, McRae AF, Bressler J, Colicino E, Hannon E, Li S et al. Meta-analysis of epigenome-wide association studies of cognitive abilities. Mol Psychiatry. 2018;23:2133-44.

29. Kaminski JA, Schlagenhauf F, Rapp M, Awasthi S, Ruggeri B, Deserno L, et al. Epigenetic variance in dopamine D2 receptor: a marker of IQ malleability? Transl Psychiatry. 2018;8:169.

30. Liu J, Siyahhan Julnes P, Chen J, Ehrlich S, Walton E, Calhoun VD. The association of DNA methylation and brain volume in healthy individuals and schizophrenia patients. Schizophr Res. 2015;169:447-52.

31. Lin D, Chen J, Ehrlich S, Bustillo JR, Perrone-Bizzozero N, Walton E, et al. Cross-tissue exploration of genetic and epigenetic effects on brain gray matter in schizophrenia. Schizophr Bull. 2018;44:443-52.

32. Freytag V, Carrillo-Roa T, Milnik A, Samann PG, Vukojevic V, Coynel D, et al. A peripheral epigenetic signature of immune system genes is linked to neocortical thickness and memory. Nat Commun. 2017;8:15193.

33. den Braber A, Bohlken MM, Brouwer RM, van 't Ent D, Kanai R, Kahn RS, et al. Heritability of subcortical brain measures: a perspective for future genome-wide association studies. Neuroimage. 2013;83:98-102.

34. Cortijo S, Wardenaar R, Colome-Tatche M, Gilly A, Etcheverry M, Labadie K, et al. Mapping the epigenetic basis of complex traits. Science. 2014;343:1145-8.

35. Sandoval J, Heyn H, Moran S, Serra-Musach J, Pujana MA, Bibikova $\mathrm{M}$, et al. Validation of a DNA methylation microarray for $450,000 \mathrm{CpG}$ sites in the human genome. Epigenetics. 2011;6:692-702.

36. Aryee MJ, Jaffe AE, Corrada-Bravo H, Ladd-Acosta C, Feinberg $\mathrm{AP}$, Hansen KD, et al. Minfi: a flexible and comprehensive bioconductor package for the analysis of Infinium DNA methylation microarrays. Bioinformatics. 2014;30:1363-9.

37. Field AP. Is the meta-analysis of correlation coefficients accurate when population correlations vary? Psychol Methods. 2005; 10:444-67.

38. Pedersen BS, Schwartz DA, Yang IV, Kechris KJ. Comb-p: software for combining, analyzing, grouping and correcting spatially correlated P-values. Bioinformatics. 2012;28:2986-8.

39. Kim MH, Choi J, Yang J, Chung W, Kim JH, Paik SK, et al. Enhanced NMDA receptor-mediated synaptic transmission, enhanced long-term potentiation, and impaired learning and memory in mice lacking IRSp53. J Neurosci. 2009;29:1586-95.

40. Chung W, Choi SY, Lee E, Park H, Kang J, Park H, et al. Social deficits in IRSp53 mutant mice improved by NMDAR and mGluR5 suppression. Nat Neurosci. 2015;18:435-43.

41. Filppula SA, Yagi AI, Kilpelainen SH, Novikov D, FitzPatrick DR, Vihinen M, et al. Delta3,5-delta2,4-dienoyl-CoA isomerase from rat liver. Molecular characterization. J Biol Chem. 1998;273:349-55.

42. Sidak Z. Rectangular confidence regions for the means of multivariate normal distributions. J Am Stat Assoc. 1967;62:626-33.

43. Benson MA, Tinsley CL, Blake DJ. Myospryn is a novel binding partner for dysbindin in muscle. J Biol Chem. 2004;279:10450-8. 
44. Durham JT, Brand OM, Arnold M, Reynolds JG, Muthukumar L, Weiler $\mathrm{H}$, et al. Myospryn is a direct transcriptional target for MEF2A that encodes a striated muscle, alpha-actinin-interacting, costamere-localized protein. J Biol Chem. 2006;281:6841-9.

45. Kielbasa OM, Reynolds JG, Wu CL, Snyder CM, Cho MY, Weiler $\mathrm{H}$, et al. Myospryn is a calcineurin-interacting protein that negatively modulates slow-fiber-type transformation and skeletal muscle regeneration. FASEB J. 2011;25:2276-86.

46. Reynolds JG, McCalmon SA, Donaghey JA, Naya FJ. Deregulated protein kinase A signaling and myospryn expression in muscular dystrophy. J Biol Chem. 2008;283:8070-4.

47. Chen X, Lee G, Maher BS, Fanous AH, Chen J, Zhao Z, et al. GWA study data mining and independent replication identify cardiomyopathy-associated 5 (CMYA5) as a risk gene for schizophrenia. Mol Psychiatry. 2011;16:1117-29.

48. Shields BJ, Jackson JT, Metcalf D, Shi W, Huang Q, Garnham AL, et al. Acute myeloid leukemia requires Hhex to enable PRC2-mediated epigenetic repression of Cdkn2a. Genes Dev. 2016;30:78-91.

49. Goodings C, Smith E, Mathias E, Elliott N, Cleveland SM, Tripathi RM, et al. Hhex is required at multiple stages of adult hematopoietic stem and progenitor cell differentiation. Stem Cells. 2015;33:2628-41.

50. Bogue CW, Zhang PX, McGrath J, Jacobs HC, Fuleihan RL. Impaired B cell development and function in mice with a targeted disruption of the homeobox gene Hex. Proc Natl Acad Sci USA. 2003;100:556-61.

51. Zhang J, McKenna LB, Bogue CW, Kaestner KH. The diabetes gene Hhex maintains delta-cell differentiation and islet function. Genes Dev. 2014;28:829-34.

52. Simpson MT, Venkatesh I, Callif BL, Thiel LK, Coley DM, Winsor KN, et al. The tumor suppressor HHEX inhibits axon growth when prematurely expressed in developing central nervous system neurons. Mol Cell Neurosci. 2015;68:272-83.

53. Sladek R, Rocheleau G, Rung J, Dina C, Shen L, Serre D, et al. A genome-wide association study identifies novel risk loci for type 2 diabetes. Nature. 2007;445:881-5.

54. Lin Z, Liu F, Shi P, Song A, Huang Z, Zou D, et al. Fatty acid oxidation promotes reprogramming by enhancing oxidative phosphorylation and inhibiting protein kinase C. Stem Cell Res Ther. 2018;9:47.

55. Wang T, Fahrmann JF, Lee H, Li YJ, Tripathi SC, Yue C, et al. JAK/STAT3-regulated fatty acid beta-oxidation is critical for breast cancer stem cell self-renewal and chemoresistance. Cell Metab. 2018;27:136-50. e135.

56. Hannon E, Lunnon K, Schalkwyk L, Mill J. Interindividual methylomic variation across blood, cortex, and cerebellum: implications for epigenetic studies of neurological and neuropsychiatric phenotypes. Epigenetics. 2015;10:1024-32.

57. Edgar RD, Jones MJ, Meaney MJ, Turecki G, Kobor MS. BECon: a tool for interpreting DNA methylation findings from blood in the context of brain. Transl Psychiatry. 2017;7:e1187.

58. Gaunt TR, Shihab HA, Hemani G, Min JL, Woodward G, Lyttleton $\mathrm{O}$, et al. Systematic identification of genetic influences on methylation across the human life course. Genome Biol. 2016;17:61.
59. Deary IJ, Gow AJ, Pattie A, Starr JM. Cohort profile: the Lothian Birth Cohorts of 1921 and 1936. Int J Epidemiol. 2012;41:1576-84.

60. McRae AF, Marioni RE, Shah S, Yang J, Powell JE, Harris SE, et al. Identification of 55,000 replicated dna methylation QTL. Sci Rep. 2018;8:17605.

61. Segre AV, Groop L, Mootha VK, Daly MJ, Altshuler D. Common inherited variation in mitochondrial genes is not enriched for associations with type 2 diabetes or related glycemic traits. PLoS Genet. 2010;6: pii: e1001058. https://doi.org/10.1371/journal. pgen. 1001058 .

62. Stranahan AM. Models and mechanisms for hippocampal dysfunction in obesity and diabetes. Neuroscience. 2015;309:125-39.

63. Fotuhi M, Do D, Jack C. Modifiable factors that alter the size of the hippocampus with ageing. Nat Rev Neurol. 2012;8:189-202.

64. Innis SM. Dietary (n-3) fatty acids and brain development. J Nutr. 2007;137:855-9.

65. Knobloch M, Braun SM, Zurkirchen L, von Schoultz C, Zamboni N, Arauzo-Bravo MJ, et al. Metabolic control of adult neural stem cell activity by Fasn-dependent lipogenesis. Nature. 2013;493:226-30.

66. Hamilton LK, Dufresne M, Joppe SE, Petryszyn S, Aumont A, Calon F, et al. Aberrant lipid metabolism in the forebrain niche suppresses adult neural stem cell proliferation in an animal model of Alzheimer's Disease. Cell Stem Cell. 2015;17:397-411.

67. Snowden SG, Ebshiana AA, Hye A, An Y, Pletnikova O, O'Brien $\mathrm{R}$, et al. Association between fatty acid metabolism in the brain and Alzheimer disease neuropathology and cognitive performance: a nontargeted metabolomic study. PLoS Med. 2017;14: e1002266.

68. Biessels GJ, Reagan LP. Hippocampal insulin resistance and cognitive dysfunction. Nat Rev Neurosci. 2015;16:660-71.

69. Raji CA, Ho AJ, Parikshak NN, Becker JT, Lopez OL, Kuller LH, et al. Brain structure and obesity. Hum Brain Mapp. 2010;31:353-64.

70. Ho AJ, Raji CA, Saharan P, DeGiorgio A, Madsen SK, Hibar DP, et al. Hippocampal volume is related to body mass index in Alzheimer's disease. Neuroreport. 2011;22:10-14.

71. Xu WL, Pedersen NL, Keller L, Kalpouzos G, Wang HX, Graff C, et al. HHEX_23 AA genotype exacerbates effect of diabetes on Dementia and Alzheimer Disease: a population-based longitudinal study. PLoS Med. 2015;12:e1001853.

72. Consortium EP. An integrated encyclopedia of DNA elements in the human genome. Nature. 2012;489:57-74.

73. Yin Y, Morgunova E, Jolma A, Kaasinen E, Sahu B, KhundSayeed $\mathrm{S}$ et al. Impact of cytosine methylation on DNA binding specificities of human transcription factors. Science. 2017;356: pii: eaaj2239. https://doi.org/10.1126/science.aaj2239.

74. Wang D, Hashimoto H, Zhang X, Barwick BG, Lonial S, Boise $\mathrm{LH}$, et al. MAX is an epigenetic sensor of 5-carboxylcytosine and is altered in multiple myeloma. Nucleic Acids Res. 2017;45:2396-407.

75. Luksys G, Ackermann S, Coynel D, Fastenrath M, Gschwind L, Heck A, et al. BAIAP2 is related to emotional modulation of human memory strength. PLoS One. 2014;9:e83707. 


\section{Affiliations}

Tianye Jia $\mathbb{D}^{1,2,3} \cdot$ Congying Chu ${ }^{1} \cdot$ Yun Liu $\mathbb{1}^{4} \cdot$ Jenny van Dongen $\mathbb{D}^{5} \cdot$ Evangelos Papastergios ${ }^{1}$. Nicola J. Armstrong ${ }^{6} \cdot$ Mark E. Bastin $^{7} \cdot$ Tania Carrillo-Roa $^{8} \cdot$ Anouk den Braber $^{5} \cdot$ Mathew Harris $\mathbb{1}^{9}$. Rick Jansen $\left(1 D^{10}\right.$ - Jingyu Liu $\mathbb{1}^{11} \cdot$ Michelle Luciano ${ }^{12}$ - Anil P. S. Ori ${ }^{13} \cdot$ Roberto Roiz Santiañez $^{14,15}$. Barbara Ruggeri ${ }^{1}$ - Daniil Sarkisyan ${ }^{16}$ - Jean Shin $1^{17} \cdot$ Kim Sungeun ${ }^{18,19}$ • Diana Tordesillas Gutiérrez ${ }^{14,20}$. Dennis van't Ent ${ }^{5}$ - David Ames ${ }^{21,22}$ - Eric Artiges ${ }^{23,24,25}$ - Georgy Bakalkin ${ }^{16}$ - Tobias Banaschewski $\mathbb{1}^{26}$. Arun L. W. Bokde ${ }^{27} \cdot$ Henry Brodaty $\mathbb{1}^{28,29}$. Uli Bromberg $\mathbb{D}^{30} \cdot$ Rachel Brouwer $^{31}$. Christian Büchel ${ }^{30}$. Erin Burke Quinlan $\mathbb{D}^{1} \cdot$ Wiepke Cahn $\mathbb{D}^{31}$ - Greig I. de Zubicaray $\mathbb{D}^{32} \cdot$ Stefan Ehrlich $^{33} \cdot$ Tomas J. Ekström $^{34}$. Herta Flor ${ }^{35,36}$ • Juliane H. Fröhner ${ }^{37}$ • Vincent Frouin $\mathbb{1}^{38}$ • Hugh Garavan ${ }^{39}$ • Penny Gowland ${ }^{40}$ - Andreas Heinz ${ }^{41,42}$. Jacqueline Hoare ${ }^{43}$ - Bernd Ittermann ${ }^{44}$. Neda Jahanshad $\mathbb{D I D}^{45}$ • Jiyang Jiang $\mathbb{D}^{28}$ - John B. Kwok ${ }^{46,47}$.

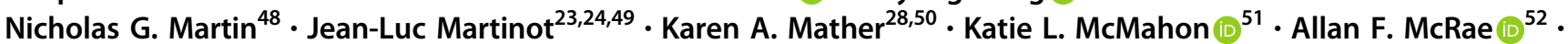
Frauke Nees ${ }^{26,35}$. Dimitri Papadopoulos Orfanos $\mathbb{1}^{38}$. Tomáš Paus ${ }^{53}$ - Luise Poustka ${ }^{54}$ - Philipp G. Sämann ${ }^{8}$. Peter R. Schofield $\mathbb{1}^{50,55}$ - Michael N. Smolka $\circledast^{37}$ - Dan J. Stein ${ }^{43,56}$ - Lachlan T. Strike ${ }^{57}$ - Jalmar Teeuw ${ }^{13,31}$. Anbupalam Thalamuthu ${ }^{28,50} \cdot$ Julian Trollor ${ }^{28,58}$ - Henrik Walter $\mathbb{1}^{41,42} \cdot$ Joanna M. Wardlaw $\mathbb{1}^{59,60} \cdot$ Wei Wen ${ }^{28}$.

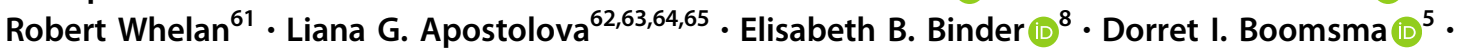
Vince Calhoun $\mathbb{D}^{11,66} \cdot$ Benedicto Crespo-Facorro ${ }^{14} \cdot \operatorname{lan} \mathrm{J}$. Deary ${ }^{12} \cdot$ Hilleke Hulshoff Pol ${ }^{31} \cdot$ Roel A. Ophoff ${ }^{31,67}$. Zdenka Pausova ${ }^{17} \cdot$ Perminder S. Sachdev $\mathbb{1}^{28,68} \cdot$ Andrew Saykin $\mathbb{1}^{69} \cdot$ Margaret J. Wright $\mathbb{1}^{48} \cdot$ Paul M. Thompson ${ }^{45}$ - Gunter Schumann $\left(\mathbb{D}^{1} \cdot\right.$ Sylvane Desrivières $^{1}$

1 Social, Genetic and Developmental Psychiatry Centre, Institute of Psychiatry, Psychology \& Neuroscience, King's College London, London, UK

2 Institute of Science and Technology for Brain-Inspired Intelligence, Fudan University, Shanghai, China

3 MOE Key Laboratory of Computational Neuroscience and BrainInspired Intelligence, Fudan University, Shanghai, China

4 MOE Key Laboratory of Metabolism and Molecular Medicine, Department of Biochemistry and Molecular Biology, School of Basic Medical Sciences, Zhongshan Hospital, Fudan University, Shanghai, China

5 Vrije Universiteit, Amsterdam, Dept Biological Psychology, Van der Boechorststraat 1, 1081 BT Amsterdam, The Netherlands

6 Mathematics and Statistics, Murdoch University, Perth, WA, Australia

7 Brain Research Imaging Centre, Centre for Clinical Brain Sciences, and Centre for Cognitive Ageing and Cognitive Epidemiology, University of Edinburgh (MEB), Edinburgh, UK

8 Department of Translational Research in Psychiatry, Max-Planck Institute of Psychiatry, Kraepelinstr, 2-10 80804

Munich, Germany

9 Centre for Clinical Brain Sciences and Edinburgh Imaging, University of Edinburgh, Edinburgh, UK

10 Department of Psychiatry, VU University Medical Centre, Amsterdam, The Netherlands

11 Department of Electrical Engineering, University of New Mexico, Albuquerque, NM, USA

12 Centre for Cognitive Ageing and Cognitive Epidemiology, Department of Psychology, University of Edinburgh, Edinburgh, UK

13 UCLA Center for Neurobehavioral Genetics, University of California, Los Angeles, Los Angeles, CA, USA
14 Department of Psychiatry, University Hospital Marqués de Valdecilla, School of Medicine, University of Cantabria, Santander, Spain

15 Centro Investigación Biomédica en Red de Salud Mental, Santander, Spain

16 Box 591, Uppsala biomedicinska centrum BMC, Husarg. 3, 751 24, Uppsala, Sweden

17 Hospital for Sick Children, University of Toronto, Toronto, ON, Canada

18 Center for Neuroimaging, Department of Radiology and Imaging Sciences, Indiana University School of Medicine, Indianapolis, IN, USA

19 Center for Computational Biology and Bioinformatics, Indiana University School of Medicine, Indianapolis, IN, USA

20 Neuroimaging Unit, Technological Facilities. Valdecilla Biomedical Research Institute IDIVAL, Santander, Cantabria, Spain

21 National Ageing Research Institute, Parkville, VIC, Australia

22 Academic Unit for Psychiatry of Old Age, University of Melbourne, St George's Hospital, Kew, VIC, Australia

23 Institut National de la Santé et de la Recherche Médicale, INSERM Unit 1000 "Neuroimaging \& Psychiatry", University Paris Sud-Paris Saclay, University Paris Descartes, Orsay, France

24 DIGITEO Labs, Gif sur Yvette, France

25 GH Nord Essonne Psychiatry Department 91G16, Orsay, France

26 Department of Child and Adolescent Psychiatry and Psychotherapy, Central Institute of Mental Health, Medical Faculty Mannheim, Heidelberg University, Square J5, 68159 Mannheim, Germany

27 Discipline of Psychiatry, School of Medicine and Trinity College 
Institute of Neuroscience, Trinity College Dublin, Dublin, Ireland

28 Centre for Healthy Brain Ageing, School of Psychiatry, University of New South Wales, Sydney, NSW, Australia

29 Dementia Centre for Research Collaboration, School of Psychiatry, University of New South Wales, Sydney, NSW, Australia

30 University Medical Centre Hamburg-Eppendorf, House W34, 3. OG, Martinistr. 52, 20246 Hamburg, Germany

31 Department of Psychiatry and Brain Center Rudolf Magnus, University Medical Center Utrecht, Utrecht, The Netherlands

32 Faculty of Health, Institute of Health and Biomedical Innovation, Queensland University of Technology, Brisbane, QLD, Australia

33 Division of Psychological and Social Medicine and Developmental Neurosciences, Faculty of Medicine, TU Dresden, Germany

34 Department of Clinical Neuroscience, Karolinska Institutet, Center for Molecular Medicine, Karolinska University Hospital, Stockholm, Sweden

35 Department of Cognitive and Clinical Neuroscience, Central Institute of Mental Health, Medical Faculty Mannheim, Heidelberg University, Square J5, Mannheim, Germany

36 Department of Psychology, School of Social Sciences, University of Mannheim, 68131 Mannheim, Germany

37 Department of Psychiatry and Neuroimaging Center, Technische Universität Dresden, Dresden, Germany

38 NeuroSpin, CEA, Université Paris-Saclay, F-91191 Gif-surYvette, France

39 Departments of Psychiatry and Psychology, University of Vermont, 05405 Burlington, VT, USA

40 Sir Peter Mansfield Imaging Centre School of Physics and Astronomy, University of Nottingham, University Park, Nottingham, UK

41 Charité-Universitätsmedizin Berlin, corporate member of Freie Universität Berlin, Humboldt-Universität zu Berlin, Berlin, Germany

42 Berlin Institute of Health, Department of Psychiatry and Psychotherapy, Campus Charité Mitte, Charitéplatz 1, Berlin, Germany

43 Department of Psychiatry and Neuroscience Institute, University of Cape Town, Cape Town, South Africa

44 Physikalisch-Technische Bundesanstalt (PTB), Braunschweig and Berlin, Berlin, Germany

45 Imaging Genetics Center, Mark and Mary Stevens Neuroimaging \& Informatics Institute, Keck School of Medicine of the University of Southern California, Marina del Rey, CA, USA

46 Central Clinical School-Brain and Mind Centre, The University of Sydney, Camperdown, NSW 2050, Australia

47 School of Medical Sciences, University of New South Wales, Sydney, NSW, Australia

48 Genetic Epidemiology, QIMR Berghofer Medical Research
Institute, Brisbane, QLD, Australia

49 Maison de Solenn, Cochin Hospital, Paris, France

50 Neuroscience Research Australia, Sydney, NSW, Australia

51 Herston Imaging Research Facility, School of Clinical Sciences, Queensland University of Technology, Brisbane, QLD, Australia

52 Institute for Molecular Bioscience, University of Queensland, Brisbane, QLD, Australia

53 Bloorview Research Institute, Holland Bloorview Kids Rehabilitation Hospital and Departments of Psychology and Psychiatry, University of Toronto, Toronto, ON M6A 2E1, Canada

54 Department of Child and Adolescent Psychiatry and Psychotherapy, University Medical Centre Göttingen, vonSiebold-Str. 5, 37075 Göttingen, Germany

55 Faculty of Medicine, University of New South Wales, Sydney, Australia

56 SAMRC Unit on Risk \& Resilience in Mental Disorders, Cape Town, South Africa

57 Queensland Brain Institute, University of Queensland, Brisbane, QLD, Australia

58 Department of Developmental Disability Neuropsychiatry, School of Psychiatry, University of New South Wales, Sydney, NSW, Australia

59 Brain Research Imaging Centre, Centre for Clinical Brain Sciences, Edinburgh Dementia Research Centre, and Centre for Cognitive Ageing and Cognitive Epidemiology, University of Edinburgh, Edinburgh, UK

60 UK Dementia Research Institute at the University of Edinburgh, Edinburgh, UK

61 School of Psychology and Global Brain Health Institute, Trinity College Dublin, Dublin, Ireland

62 Department of Neurology, Indiana University School of Medicine, Indianapolis, IN, USA

63 Department of Radiology and Imaging Sciences, Indiana University School of Medicine, Indianapolis, IN, USA

64 Department of Medical and Molecular Genetics, Indiana University School of Medicine, Indianapolis, IN, USA

65 Indiana Alzheimer Disease Center, Indiana University School of Medicine, Indianapolis, IN, USA

66 Tri-institutional Center for Translational Research in Neuroimaging and Data Science (TReNDS), Emory University, 30303, Atlanta, GA, USA

67 Semel Institute for Neuroscience and Human Behavior, University of California, Los Angeles, Los Angeles, CA, USA

68 Neuropsychiatric Institute, Prince of Wales Hospital, Sydney, NSW, Australia

69 Radiology and Imaging Sciences, Indiana University School of Medicine, Indianapolis, IN 46202, USA 